\title{
Prediction of the Length of Intensive Care After Cardiac Surgery Under Cardiopulmonary Bypass: Logistic Regression Analysis Based on Troponin I And EuroSCORE II
}

\author{
Tai Lai Ti.Tai Wan Gu, ${ }^{1}$ Jianjiang $W u,{ }^{2}$ Haiting $Z$ Zhan, ${ }^{2}$ Yidan Huang, ${ }^{2}$ Jiang Wang ${ }^{2}$ \\ ${ }^{1}$ The First Clinical Institute of Xinjiang Medical University, Urumqi, China; \\ ${ }^{2}$ Anesthesiology Department of The First Affiliated Hospital of Xinjiang Medical University, Urumqi, Xinjiang, China
}

\section{ABSTRACT}

Aim: This study is to establish a model for patients undergoing cardiac surgery under cardiopulmonary bypass $(\mathrm{CPB})$ to predict the length of intensive care.

Methods: This is a single center retrospective study. A total of 265 patients admitted to the ICU after CPB from 2016 to 2017 were enrolled in the study. Preoperative indicators, intraoperative parameters, and postoperative data were collected. Each patient was scored for EuroSCORE II before surgery. According to the length of intensive care, all patients were divided into two groups: short stay $(<72 \mathrm{~h})$ and long stay $(\geq 72 \mathrm{~h}$ ). A binary logistic regression analysis was performed to establish a regression model to evaluate the predictive performance of the indicators and the EuroSCORE II scoring system on the length of the intensive care.

Results: Both troponin I and EuroSCORE II could predict the length of intensive care of patients undergoing cardiac surgery under CPB. After combing the two factors, the prediction efficiency was higher. Comparing the prediction results with the actual data, it showed that the method had high overall accuracy.

Conclusions: The predictive model based on cTnI and EuroSCORE II can accurately predict the length of intensive care of patients undergoing cardiac surgery under $\mathrm{CPB}$. This predictive model may help to improve ICU resource management.

\section{INTRODUCTION}

The bed utilization rate in the intensive care unit (ICU) is the determined by the turnover of patients in the cardiac surgery department. Therefore, an accurate statistical tool can make the patient's operation schedule more reasonable and provide a reference for the hospital to improve the bed

Received April 25, 2021; accepted fune 7, 2021.

Correspondence: Fiang Wang, No.137 of Li Yushan Road, Urumqi City, 830054, Xinjiang, China (e-mail: 710985359@qq.com). utilization rate [Meadows 2018]. Patients in ICU requiring prolonged hospital stays account for a large proportion [Wagener 2011]. The cost of intensive care, coupled with the financial requirements of cardiac surgery itself, makes the preoperative evaluation method particularly important [Andersen 2015]. Most scoring systems have been validated in the general intensive care patients, but not in specific patients like those undergoing cardiac surgery [Exarchopoulos 2015]. Therefore, the pathophysiological changes caused by extracorporeal circulation and related invasive procedures (IABP, hemodialysis, ECMO, and mechanical ventilation, etc.) may not be covered [Hekmat 2005; Doerr 2011; Weiss 2000].

EuroSCORE II is a commonly used risk assessment system for cardiac surgery to predict short-term mortality after cardiac surgery [Nashef 1999]. Previous study has used EuroSCORE II to predict the length of postoperative intensive care and the probability of postoperative complications [Meadows 2018]. In addition, cardiac biomarkers also have been used to assess the risk of cardiac surgery [Mukaida 2020]. Cardiac Troponin I (cTnI) is recognized as an important indicator for evaluating myocardial injury and generally is used to predict the development and mortality of patients with cardiovascular diseases [van Geene 2010; Gahl 2018]. Whether cTnI could provide additional predictive information to EuroSCORE II still is unclear. Additionally, the predictive effect of cTnI on the length of intensive care of patients undergoing cardiac surgery under cardiopulmonary bypass $(\mathrm{CPB})$ has not been reported. Therefore, this study is to establish a model for patients undergoing cardiac surgery under cardiopulmonary bypass $(\mathrm{CPB})$ to predict the length of intensive care.

\section{METHODS}

Patients: A total of 265 patients admitted to the ICU after CBP from 2016 to 2017 were enrolled. The clinical data of patients before and during operation, and during intensive care were collected and retrospectively analyzed.

The inclusion criteria were as follows: Patients received cardiac surgery under CBP, then the patients were transferred to ICU for intensive care after surgery and to the general ward for consolidation after the condition was stable until discharge. The exclusion criteria were patients undergoing off-pump surgery and those with missing data or who died 
in hospital. Written informed consent could be avoided, as this was a retrospective study. The study was approved by the ethics review board of Xinjiang Medical University.

Preoperative data collection: Demographic data, including sex, age, height, weight, body mass index, comorbidities, risk level indicators, and preoperative ultrasound examination results were collected. New logistic EuroSCORE II is calculated, according to the official EuroSCORE II scoring system (http://www.euroscore.org/calc.html). The blood biochemical results and biomarker test results before the surgery also were collected, including white blood cells, hemoglobin, platelets, creatinine, glucose, cTnI, potassium ions, sodium ions, prothrombin time, and prothrombin time international standardized ratio, etc.

Anesthesia: Anesthesia was induced with midazolam, fentanyl, and rocuronium. Before CPB, sevoflurane was inhaled for anesthesia, while propofol and fentanyl were given to maintain anesthesia. At the end of the operation, fentanyl was discontinued, and propofol was continuously given until extubation in the ICU.

CPB management: Modified del Nido blood (4:1) cardioplegia $20 \mathrm{~mL} / \mathrm{kg}$ was used, another $10 \mathrm{~mL} / \mathrm{kg}$ was added one hour later, and this dose was used for infusion every half hour. The aortic pump flow rate was controlled at 60-100 $\mathrm{mL} / \mathrm{kg} / \mathrm{min}$, the average arterial pressure was controlled at $50-80 \mathrm{mmHg}$, and the arterial blood oxygen saturation was controlled at more than $90 \%$. The suspension of red blood cells was given when the hematocrit was less than $20 \%$.

Surgical procedures: All operations using CPB, including coronary artery bypass grafting, valve replacement, aortic surgery, and surgical treatment of adult congenital heart disease were included in this study. Intraoperative variables, including the $\mathrm{CPB}$ time, lowest intraoperative hemoglobin, and lowest hematocrit, etc. were evaluated.

Clinical outcomes: The primary outcome measurement was whether the length of the intensive care was prolonged. Seventy-two hours (three days) was set as the cut-off point for determining whether the intensive care time was prolonged. In addition to medical factors, non-medical factors, such as the size of the hospital ICU, doctor's opinion, feasibility of returning to the general ward, the busy surgical schedule, and particularity of the specific time schedule for the day (generally after early rounds), etc. [Messaoudi 2009], also affects intensive care time. On the other hand, Mazer et al. reported that the average ICU hospital stay after cardiac surgery was 2.3 to 5.2 days [Mazer 1993]. Pascal et al. reported that the optimal cut-off value of intensive care time of elderly people over 80 years old after cardiac surgery was four days, and $84 \%$ of the patients without postoperative complications had an intensive care time less than four days [Augustin 2016].

The percentage of prolonged intensive care time was determined based on the hospital's electronic medical record system. The secondary outcome measurements were the intubation time, postoperative suction drainage, and total hospital stay.

Statistical analysis: The data was analyzed using SPSS 25.0 (IBM Inc. US). The sample size was estimated by the multivariate binary logistic regression analysis, based on the positive rate of outcome variables and number of independent variables included in the multivariate regression analysis. Continuous variables of normal distribution were presented as mean \pm standard deviation; those of non-normal distribution were expressed as median and interquartile range. Categorical variables were expressed as n (percentage). As cTnI belonged to a positive skewed distribution, $\log 10$ transformation was performed on $\mathrm{c} T \mathrm{n} \mathrm{n}$ before regression analysis.

Univariate binary logistic regression analysis was used to comprehensively evaluate the relationship between each variable and the length of intensive care. Due to the limited sample size, only a limited multi-factor regression model was allowed, therefore, the significance requirement was taken as $P<0.005$ [Cuthbertson 2009]. Subsequently, multivariate logistic regression analysis was performed, and regression models with cTnI were established, according to the categories of the candidate factors (demographic data, preoperative tests, operative variable, risk stratification, etc.). Whether each candidate factor and $\mathrm{cTnI}$ were independent predictors and whether the prediction efficiency was improved after modeling were observed.

The receiver operating characteristic curve (ROC) was used to assess the correlation between the predictor and the length of intensive care, and to determine the cutoff value of the predictor. Univariate binary logistic regression analysis method was used to evaluate the correlation between the prolonged intensive care time and the cutoff value of the predictor, and $\mathrm{r}^{2}$ and corresponding $P$ value were calculated, respectively. Chi-square test was used to analyze the correlation of predictors, intubation time, hospital stay, and intensive care time between the two groups. The odds ratio and $95 \%$ confidence interval $(95 \% \mathrm{CI})$ were calculated. A $P$ value of less than 0.05 was considered as statistically significant.

\section{RESULTS}

Proportion of prolonged intensive care time: This study analyzed the data of 265 patients undergoing cardiac surgery with CPB. There were 159 males and 106 females. The average age was $46.15 \pm 11.69$ years old. The average BMI was $24.8 \pm 4.1$. The average length of ICU care was $52.5 \pm 61.4$ hours. The average of the length of total hospital stay was 13.8 7.3 days. Among them, 50 patients had prolonged intensive care time, accounting for about $18.9 \%$.

Prediction of prolonged intensive care time based on preoperative variables: Univariate logistic regression analysis of preoperative indexes and prolonged intensive care time are shown in Table 1 and Table 2. (Table 1) (Table 2) The results of the $c h i$-square test and logistic regression analysis of the variables related to the operation are shown in Table 3. (Table 3) EuroSCORE II, sex, weight, height, LVEF (left ventricular ejection fraction), cTnI, and creatinine were highly correlated with prolonged intensive care time $(P<0.05)$.

cTnI in the prediction of intensive care time: $\mathrm{c} T n I$ was significantly correlated with the length of intensive care time $(P<0.005)$. The cTnI and EuroSCORE II were independent risk factors for each other $(P<0.005$. (Table 4) 
Table 1. Preoperative variables related to demography and echocardiography and results of univariate logistic regression analyses for prolonged intensive care

\begin{tabular}{|c|c|c|c|c|c|c|}
\hline Age & $47(38.5-55)$ & $46(37-54)$ & $44.5(52-57)$ & $1.041(1.011-1.072)$ & 0.048 & 0.007 \\
\hline Body weight $(\mathrm{kg})$ & $70(60-80)$ & $68(58-79)$ & $76.5(66.75-86.25)$ & $1.038(1.015-1.061)$ & 0.068 & $0.001 *$ \\
\hline \multicolumn{7}{|l|}{ Comorbidities } \\
\hline Hypertension & $61(23.0 \%)$ & $42(68.9 \%)$ & $19(31.1 \%)$ & $0.396(0.204-0.769)$ & 0.430 & 0.006 \\
\hline Diabetes & $11(4.2 \%)$ & $6(54.5 \%)$ & $5(45.5 \%)$ & $3.870(1.132-13.238)$ & 0.026 & 0.031 \\
\hline Chronic obstructive lung disease & $2(0.8 \%)$ & $2(100 \%)$ & 0 & 0 & 0 & 0.999 \\
\hline Previous cardiac surgery & $4(1.5 \%)$ & $3(75 \%)$ & $1(25 \%)$ & $1.442(0.147-14.162)$ & 0.001 & 0.753 \\
\hline EuroSCORE II (\%) & $1.06(0.69-1.435)$ & $0.98(0.69-1.33)$ & $1.24(0.74-2.24)$ & $1.660(1.245-2.213)$ & 0.100 & $0.001 *$ \\
\hline \multicolumn{7}{|l|}{ Echocardiography data } \\
\hline LVEF & $63(58-66)$ & $59(63-67)$ & $59(54-63.25)$ & $0.918(0.879-0.959)$ & 0.095 & 0.001 * \\
\hline
\end{tabular}

Data are presented as median and interquartile range or numbers (\%). Results of the logistic regression are given as odds ratio with $95 \%$ confidence interval $(\mathrm{Cl})$ and Nagelkerke's generalized $r^{2}\left(r^{2}\right)$. LVEF, left ventricular ejection fraction; NYHA, New York Heart Association functional class

Table 2. Preoperative biomarkers and results of univariate logistic regression analyses for prolonged intensive care - Associations with prolonged intensive care

\begin{tabular}{|c|c|c|c|c|c|c|}
\hline Hemoglobin $(\mathrm{g} / \mathrm{dL})$ & $13.8(12.7-14.95)$ & $13.7(12.7-14.7)$ & $14.3(12.95-15.33)$ & 1.011 (0.957-1.067) & 0.001 & 0.701 \\
\hline Platelet count $(104 / \mu \mathrm{L})$ & $225(181-258.5)$ & $229(183-261)$ & $203.5(170.5-247.3)$ & $0.994(0.989-1.000)$ & 0.028 & 0.039 \\
\hline Creatinine $(\mu \mathrm{mol} / \mathrm{L})$ & $68.0(57.0-80.5)$ & $55.0(66.0-78.0)$ & $74(63.30-88.4)$ & $1.029(1.013-1.045)$ & 0.083 & $<0.001 *$ \\
\hline Sodium (mmol/L) & $140(138-142)$ & $140(138-143)$ & $140(137.9-142)$ & $1.006(0.962-1.052)$ & 0.000 & 0.796 \\
\hline Potassium (mmol/L) & $4.0(3.7-4.3)$ & $4.0(3.7-4.3)$ & $3.9(3.7-4.1)$ & $0.513(0.219-1.200)$ & 0.018 & 0.124 \\
\hline Glucose & $4.8(4.4-5.3)$ & $4.8(4.4-5.2)$ & $4.9(4.5-5.7)$ & $1.248(0.983-1.586)$ & 0.019 & 0.069 \\
\hline
\end{tabular}

Data are presented as median and interquartile range. Results of the logistic regression are given as odds ratio with $95 \%$ confidence interval $(\mathrm{Cl})$ and $\mathrm{Nagelker-}$ ke's generalized $r^{2}\left(r^{2}\right)$. APTT, activated partial thromboplastin time; PT-INR, international normalized ratio of prothrombin time; cTnl, cardiac troponin I 
Table 3. Operative variables and results of the Chi square test and univariate logistic regression analyses for prolonged intensive care - Associations with prolonged intensive care

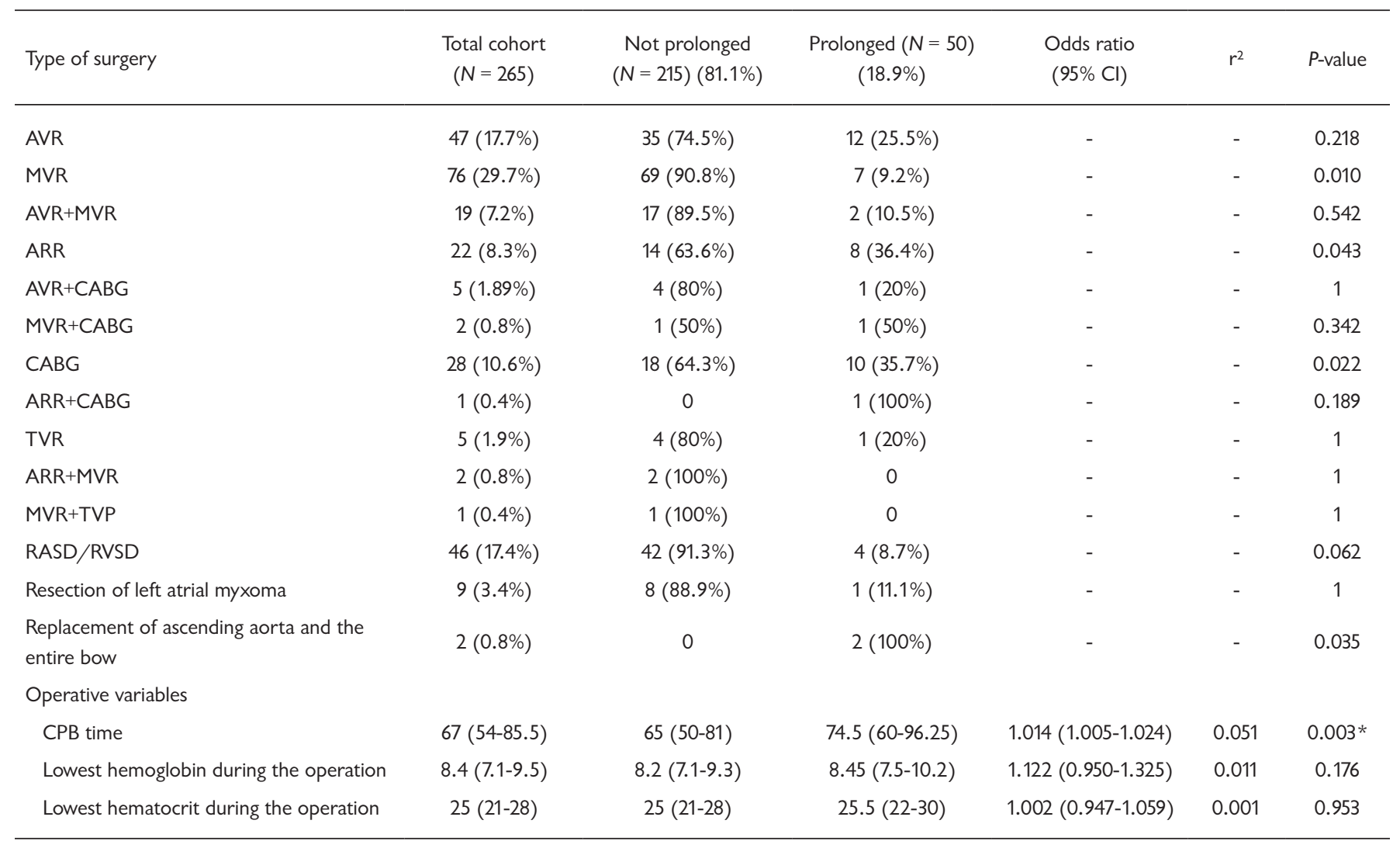

Data are presented as median and interquartile range or numbers (\%). Results of the logistic regression are given as odds ratio with $95 \%$ confidence interval $(\mathrm{Cl})$, and Nagelkerke's generalized $\mathrm{r}^{2}\left(\mathrm{r}^{2}\right)$. AAR, ascending aortic replacement; ARR, aortic root replacement; AVR/P, aortic valve replacement or plasty; CABG, coronary artery bypass graft; CPB, cardiopulmonary bypass; MVR/P, mitral valve replacement or plasty; TVP, tricuspid valve plasty; RASD/RVSD, repair of atrial/ventricular septal defect

Table 4. Results of multivariate logistic regression analysis for prolonged intensive care

\begin{tabular}{lcl}
\hline Variables & Odds ratio (95\%) & $P$-value \\
\hline Log(cTnl) & $2.591(1.463-5.590)$ & $0.001 *$ \\
EuroSCORE II & $1.566(1.188-2.064)$ & $0.001 *$ \\
Overall model fit & $r^{2}=0.164$ & $<0.001$ \\
\hline
\end{tabular}

Results of the logistic regression are given as odds ratio with $95 \%$ confidence interval $(\mathrm{Cl})$, and Nagelkerke's generalized $r^{2}\left(r^{2}\right)$.

Relationship between cTnI and established risk assessment system for cardiac surgery: Model 5 in Table 4 showed that $\mathrm{c} T \mathrm{nI}$ and the existing cardiac risk assessment system were independent in predicting whether the intensive care time is prolonged. The Nagelkerke's generalized $\mathrm{r}^{2}\left(\mathrm{r}^{2}=\right.$ $0.164)$ and the correct prediction percentage $(83.6 \%)$ of the two established models were greater than those of $\mathrm{cTnI}\left(\mathrm{r}^{2}=\right.$
0.089, 81.9\%) alone and EuroSCORE II $\left(\mathrm{r}^{2}=0.1,83 \%\right)$ alone. This suggests that the addition use of $\mathrm{c} T \mathrm{nI}$ can improve the predictive ability of EuroSCORE II.

Cutoff values of cTnI and EuroSCORE II: ROC analysis assessing the association between each of $\log (\mathrm{cTnI}), \mathrm{CPB}$ time, EuroSCORE II, creatinine, and prolonged intensive care time are shown in Figure 1. (Figure 1) The cutoff values were 1.27 for $\log c \operatorname{TnI}$ (corresponding to cTnI, 18.9) and $1.065 \%$ for EuroSCORE II.

Relationship of cTnI and EuroSCORE II with intensive care time and other outcome variables: The two predictors of cTnI and EuroSCORE II were converted into binary variables based on the cut-off value, and binary logistic regression analysis was performed to determine whether the intensive care time was prolonged. The results showed that $\mathrm{cTnI} \geq 18.9$ and EuroSCORE II $\geq 1.065 \%$ were significantly correlated with the intensive care time, and the combination of the two was related to the further increase of the intensive care time. (Table 5) In addition, $c T n I \geq 18.9$ and EuroSCORE II $\geq 1.065 \%$ were both related to the increased drainage volume and increased ventilation time of endotracheal intubation at $24 \mathrm{~h}$ after operation. (Table 6) 


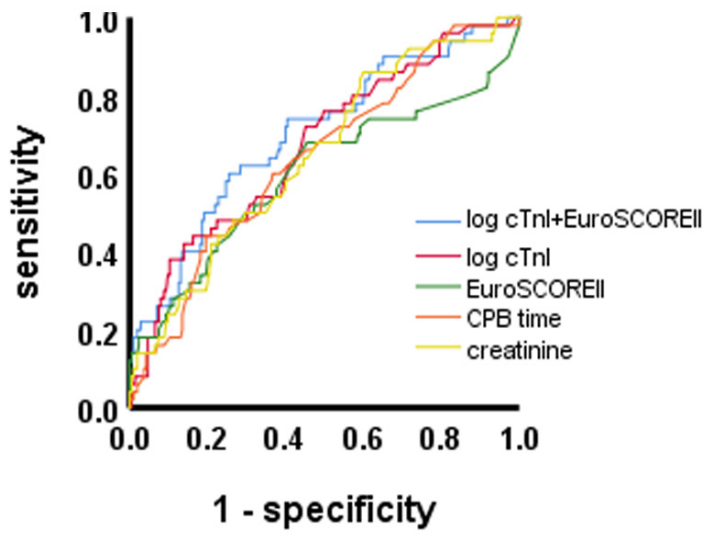

Figure 1. Results of the receiver operating characteristic (ROC) analysis assessing the association between each of Log(cTnl), CPB time, EuroSCORE II, creatinine, Log(cTnl)+EuroSCORE II and the prolonged intensive care time.

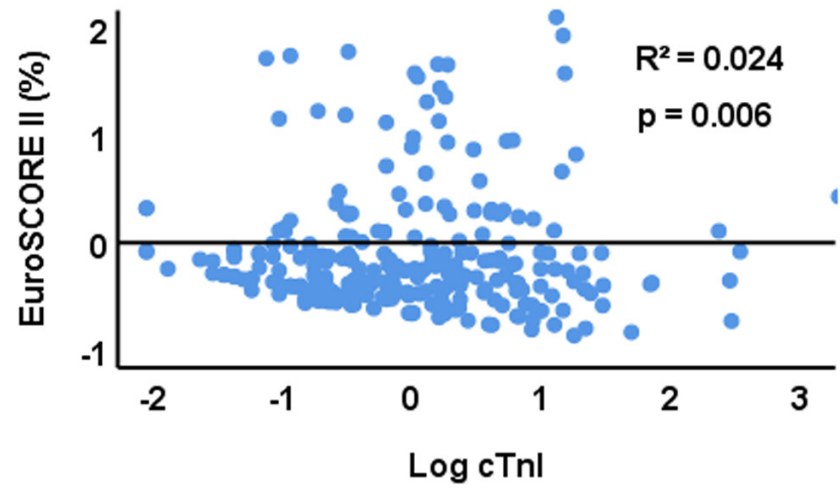

Figure 2. The linear regression analysis of the relationship between log cTnl and EuroSCORE II.

Table 5. Effects of high log cTnl and/or high EuroSCORE II on actual prolonged intensive care rates and predicted increases prolonged intensive care

\begin{tabular}{|c|c|c|c|c|}
\hline $\begin{array}{l}\text { Variables } \\
\text { CTnl } \geq 18.9\end{array}$ & \multicolumn{2}{|c|}{$\begin{array}{l}\text { Actual proportion of prolonged intensive care } \\
\text { Odds ratio }\end{array}$} & \multicolumn{2}{|c|}{ Results of the logistic regression analyses } \\
\hline EureSCORE II $\geq 1.065 \%$ & $25.8 \%(34 / 132)$ & $2.537(1.322-4.869)$ & 0.050 & $0.005^{*}$ \\
\hline CTnI $\geq 18.9$ \& EuroSCORE $\| \geq 1.065 \%$ & $53.1(17 / 32)$ & $6.869(3.130-15.073)$ & 0.130 & $<0.001 *$ \\
\hline
\end{tabular}

Results of the univariate binary logistic regression analysis to evaluate effects of high cTnl and/or high EuroSCORE II according to cutoff values of log cTnl $(1.27 \mathrm{ng} / \mathrm{L})$ corresponding to a cTnl value(18.9ng/L) and EuroSCORE II (1.065\%) on prolonged intensive care are given as odds ratio with $95 \%$ confidence interval $(\mathrm{Cl})$ and Nagelkerke's generalized $r^{2}\left(r^{2}\right)$. Actual prolonged intensive care in patients with high cTnl and/or high EuroSCORE II also are shown. See Figure 1 for cutoff values of cTnl and EuroSCORE II.

Table 6. Effects of cTnl and/or EuroSCORE II dichotomized according to cutoff values on Postoperative suction drainage more than $1000 \mathrm{ml}$ and Prolonged ventilation longer than $24 \mathrm{~h}$

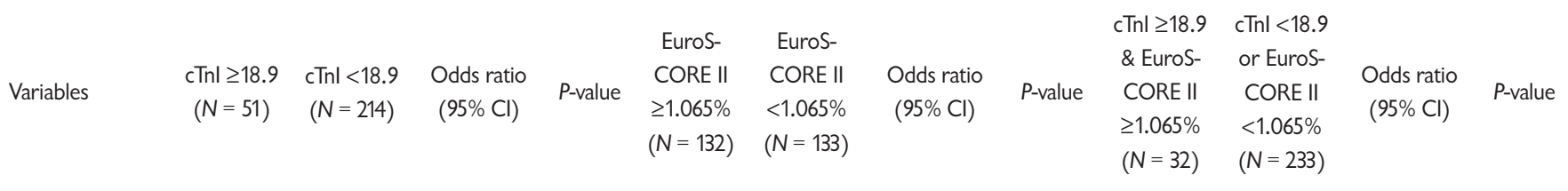

\begin{tabular}{|c|c|c|c|c|c|c|c|c|c|c|c|c|}
\hline $\begin{array}{l}\text { Postoperative } \\
\text { suction drainage } \\
\text { more than } \\
1000 \mathrm{ml}\end{array}$ & $5(9.8)$ & $5(2.3)$ & $\begin{array}{c}4.54 \\
(1.26-16.34)\end{array}$ & $0.012 *$ & $9(6.8)$ & $1(0.8)$ & $\begin{array}{c}9.66 \\
(1.21-77.35)\end{array}$ & $0.010 *$ & $5(15.6)$ & $5(2.1)$ & $\begin{array}{c}8.44 \\
(2.3-31.1)\end{array}$ & $0.000 *$ \\
\hline $\begin{array}{l}\text { Prolonged } \\
\text { ventilation } \\
\text { longer than } 24 \mathrm{~h}\end{array}$ & $15(29.4)$ & $32(15)$ & $\begin{array}{c}2.37 \\
(1.17-4.82)\end{array}$ & $0.015 *$ & $30(22.7)$ & $17(12.8)$ & $\begin{array}{c}2.0 \\
(1.04-3.85)\end{array}$ & $0.034 *$ & $11(34.3)$ & $36(15.5)$ & $\begin{array}{c}2.87 \\
(1.27-6.45)\end{array}$ & $0.009 *$ \\
\hline
\end{tabular}

Categorical data are presented as n (\%). Variables were analyzed with Chi square test. See also Figure 1 for cutoff values of log cTnl and EuroSCORE II. Newly required dialysis is divided into two types. 
Relationship between cTnI and EuroSCORE II: The linear regression analysis showed that $\log (\mathrm{c} T \mathrm{Tn})$ was significantly correlated with EuroSCORE II $(P=0.006)$, but the adjusted $\mathrm{r}^{2}$ was 0.024 , indicating the evaluation of the linear regression model was not statistically significant. (Figure 2)

\section{DISCUSSION}

The results of this study showed that cTnI could independently predict whether the intensive care time would be prolonged after cardiac surgery using CPB. The existing cardiac surgery risk assessment system EuroSCORE II also had good prediction accuracy. The additional use of cTnI can improve the prediction accuracy of EuroSCORE II. This process was independent of CPB time, LVEF, and creatinine value. In addition, the increase of cTnI and EuroSCORE II was related to the postoperative increased drainage volume and ventilation time.

Previous study showed that $\mathrm{cTnI}$ was correlated with the prognosis and mortality in patients who underwent cardiac surgery [Muehlschlegel 2010], and the basis for the prediction of postoperative mortality using $\mathrm{cTnI}$ has been provided [van Geene 2010]. However, the prediction of intensive care time by cTnI is less investigated. Our study found that cTnI could predict whether the intensive care period of patients undergoing cardiac surgery using CBP would be prolonged. In addition, the increase of cTnI was related with some direct factors, which prolong the intensive care time, such as increased drainage volume and ventilation time. This may explain why cTnI can predict the length of intensive care time.

EuroSCORE II, as an existing surgical risk assessment system for patients treated with cardiac surgery, is commonly used to predict mortality within 30 days after cardiac surgery [Nashef 2012]. The results of this study showed that EuroSCORE II could also predict whether the intensive care time is prolonged. In addition, EuroSCORE II was related to increased drainage volume and ventilation time.

A previous large-scale study, involving 18,377 patients, showed that EuroSCORE II can not only accurately predict postoperative mortality, but also predict prolonged intensive care time and the occurrence rate of major complications [Meadows 2018], which is consistent with our results. However, the effect of CPB on patient prognosis was not evaluated. The current not only used EuroSCORE II to predict the intensive care time of patients undergoing cardiac surgery using $\mathrm{CPB}$, but also provided a new direction for the prediction using EuroSCORE II. The results of this study showed that both $c \operatorname{TnI} \geq 18.9$ and EuroSCORE II $\geq 1.065 \%$ were related to the prolonged ventilation time and increased postoperative drainage. This also may suggest that $\mathrm{c} T \mathrm{nI}$ and EuroSCORE II may predict the occurrence of postoperative complications.

Although previous studies have shown that cTnI and EuroSCORE II can predict postoperative mortality [van Geene 2010; Nashef 2012] and the additional use of cTnI can improve the predictive performance [Fellahi 2011], there is no report on the predictive value of $\mathrm{cTnI}$ and EuroSCORE II on the length of intensive care time. In this study, we found that the prediction of $\mathrm{c} T \mathrm{Tn}$ for the length of intensive care time was independent of EuroSCORE II, and the linear relationship between the two factors was very weak. Moreover, cTnI could improve the predictive performance of EuroSCORE II. The existing cardiac surgery risk assessment system contains only one biomarker, creatinine, or creatinine clearance. LVEF also is included in the EuroSCORE II scoring system, but it is not a reliable indicator of cardiac function in patients with heart valve disease [Galli 2014; Ishigaki 2020]. The current study also showed that the AUC area of LVEF was less than 0.5 , and the diagnostic value was much less than cTnI. Therefore, cTnI may be more objective than LVEF to reflect the state of heart disease and improve the practicality of the existing cardiac surgery risk assessment system.

Study limitation: This study has some limitations. First, the sample size was relatively small. Second, this is a singlecenter study. Further multi-center studies with larger sample size are warranted. Third, we only included Chinese patients. The situation might be different with other ethnicities.

\section{CONCLUSION}

Our findings support the use of preoperative $\mathrm{cTnI}$ and the established cardiac surgery risk assessment system EuroSCORE II to identify the patients with increased intensive care time, prolonged endotracheal intubation ventilation time, and increased risk of increased drainage volume after cardiac surgery using CBP. CTnI and EuroSCORE II are independent of each other in predicting whether intensive care time is prolonged, moreover they are independent of CPB time and patient's preoperative creatinine value. The additional use of cTnI can improve the predictive performance of EuroSCORE II and provide important information for the risk assessment tool of cardiac surgery.

\section{REFERENCES}

Andersen LW, Holmberg MJ, Doherty M, Khabbaz K, Lerner A, Berg KM, Donnino MW. 2015. Postoperative Lactate Levels and Hospital Length of Stay After Cardiac Surgery. J Cardiothorac Vasc Anesth. 29: 1454-1460.

Augustin P, Tanaka S, Chhor V, Provenchère S, Arnaudovski D, Ibrahim H, Dilly MP, Allou N, Montravers P, Philip I. 2016. Prognosis of Prolonged Intensive Care Unit Stay After Aortic Valve Replacement for Severe Aortic Stenosis in Octogenarians. J Cardiothorac Vasc Anesth. 30: $1555-1561$.

Cuthbertson BH, Croal BL, Rae D, Gibson PH, McNeilly JD, Jeffrey RR, Smith WC, Prescott GJ, Buchan KG, El-Shafei H, Gibson GA, Hillis GS. 2009. N-terminal pro-B-type natriuretic peptide levels and early outcome after cardiac surgery: a prospective cohort study. $\mathrm{Br} \mathrm{J}$ Anaesth. 103: 647-653.

Doerr F, Badreldin AM, Heldwein MB, Bossert T, Richter M, Lehmann T, Bayer O, Hekmat K. 2011. A comparative study of four intensive care outcome prediction models in cardiac surgery patients. J Cardiothorac Surg. 6: 21 . 
Exarchopoulos T, Charitidou E, Dedeilias P, Charitos C, Routsi C. 2015. Scoring Systems for Outcome Prediction in a Cardiac Surgical Intensive Care Unit: A Comparative Study. Am J Crit Care. 24: 327-334; quiz 335.

Fellahi JL, Le Manach Y, Daccache G, Riou B, Gérard JL, Hanouz JL. 2011. Combination of EuroSCORE and cardiac troponin I improves the prediction of adverse outcome after cardiac surgery. Anesthesiology. 114: 330-339.

Gahl B, Göber V, Odutayo A, Tevaearai Stahel HT, da Costa BR, Jakob SM, Fiedler GM, Chan O, Carrel TP, Jüni P. 2018. Prognostic Value of Early Postoperative Troponin T in Patients Undergoing Coronary Artery Bypass Grafting. J Am Heart Assoc 7.

Galli E, Lancellotti P, Sengupta PP, Donal E. 2014. LV mechanics in mitral and aortic valve diseases: value of functional assessment beyond ejection fraction. JACC Cardiovasc Imaging. 7: 1151-1166.

Hekmat K, Kroener A, Stuetzer H, Schwinger RH, Kampe S, Bennink GB, Mehlhorn U. 2005. 7 Daily assessment of organ dysfunction and survival in intensive care unit cardiac surgical patients. Ann Thorac Surg. 9: $1555-1562$

Ishigaki T, Shingu Y, Katoh N, Wakasa S, Katoh H, Ooka T, Kubota S, Matsui Y. 2020. Perioperative changes of the slope in the preload recruitable stroke work relationship by a single-beat technique after mitral valve surgery in functional mitral regurgitation with non-ischemic dilated cardiomyopathy. Gen Thorac Cardiovasc Surg. 68: 30-37.

Mazer CD, Byrick RJ, Sibbald WJ, Chovaz PM, Goodman SJ, Girotti MJ, Hall JK, Pagliarello J. 1993. Postoperative utilization of critical care services by cardiac surgery: a multicenter study in the Canadian healthcare system. Crit Care Med. 21: 851-859.

Meadows K, Gibbens R, Gerrard C, Vuylsteke A. 2018. Prediction of Patient Length of Stay on the Intensive Care Unit Following Cardiac Surgery: A Logistic Regression Analysis Based on the Cardiac Operative Mortality Risk Calculator, EuroSCORE. J Cardiothorac Vasc Anesth. 32: 2676-2682.
Messaoudi N, De Cocker J, Stockman B, Bossaert LL, Rodrigus IE. 2009. Prediction of prolonged length of stay in the intensive care unit after cardiac surgery: the need for a multi-institutional risk scoring system. J Card Surg. 24: 127-133.

Muehlschlegel JD, Perry TE, Liu KY, Fox AA, Collard CD, Shernan SK, Body SC. 2010. Heart-type fatty acid binding protein is an independent predictor of death and ventricular dysfunction after coronary artery bypass graft surgery. Anesth Analg. 111: 1101-1109.

Mukaida H, Hayashida M, Matsushita S, Endo D, Oishi A, Shimada A, Hata H, Kajimoto K, Yamamoto T, Amano A. 2020. Free triiodothyronine (fT3) and B-type natriuretic peptide (BNP) predict in-hospital mortality after valve surgery. Gen Thorac Cardiovasc Surg. 68: 585-595.

Nashef SA, Roques F, Michel P, Gauducheau E, Lemeshow S, Salamon R. 1999. European system for cardiac operative risk evaluation (EuroSCORE). Eur J Cardiothorac Surg. 16: 9-13.

Nashef SA, Roques F, Sharples LD, Nilsson J, Smith C, Goldstone AR, Lockowandt U. 2012. EuroSCORE II. Eur J Cardiothorac Surg. 41: 734744; discussion 744-735.

van Geene Y, van Swieten HA, Noyez L. 2010. Cardiac troponin I levels after cardiac surgery as predictor for in-hospital mortality. Interact Cardiovasc Thorac Surg. 10: 413-416.

Wagener G, Minhaz M, Wang S, Panzer O, Wunsch H, Playford HR, Sladen RN. 2011. The Surgical Procedure Assessment (SPA) score predicts intensive care unit length of stay after cardiac surgery. J Thorac Cardiovasc Surg. 142: 443-450.

Weiss YG, Merin G, Koganov E, Ribo A, Oppenheim-Eden A, Medalion B, Peruanski M, Reider E, Bar-Ziv J, Hanson WC, Pizov R. 2000. Postcardiopulmonary bypass hypoxemia: a prospective study on incidence, risk factors, and clinical significance. J Cardiothorac Vasc Anesth. 14: 506-513. 